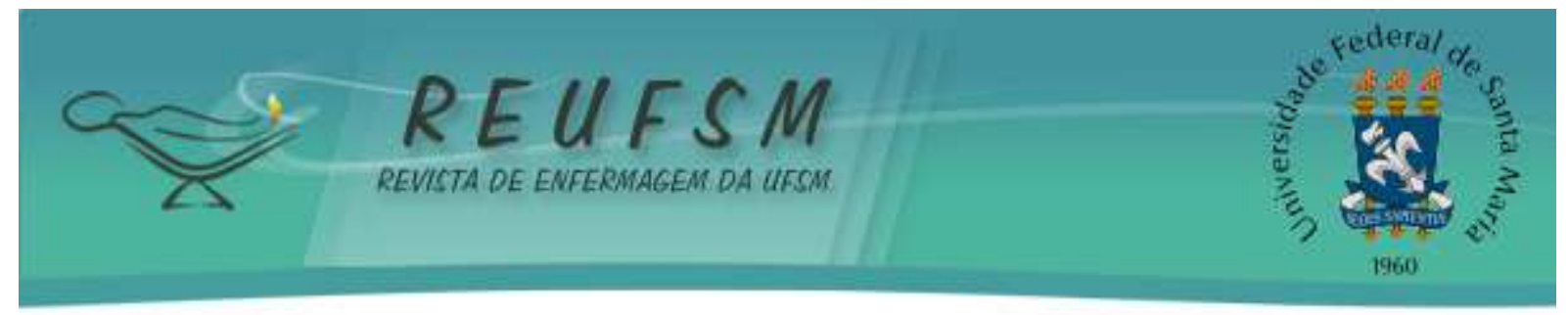

\title{
RELAÇÕES SOCIAIS INFORMAIS EM IDOSO COM HIPERTENSÃO E/OU DIABETES
}

\author{
INFORMAL SOCIAL RELATIONSHIP REGARDING ELDERLY WITH \\ HYPERTENSION AND/OR DIABETES
}

\section{RELACIONES SOCIALES INFORMALES EN IDOSOS CON HIPERTENSIÓN Y/O DIABETES}

Doi: $10.5902 / 2179769227800$

\author{
Mariangela Uhlmann Soares ${ }^{1}$ \\ Bruno Pereira Nunes ${ }^{2}$ \\ Louriele Soares Wachs ${ }^{3}$ \\ Marciane Kessler ${ }^{4}$ \\ Alitéia Santiago Dilélio ${ }^{5}$ \\ Deisi Cardoso Soares ${ }^{6}$ \\ Luiz Augusto Facchini ${ }^{7}$ \\ Elaine Thumé ${ }^{8}$
}

RESUMO: Objetivo: avaliar as relações sociais informais dos idosos com diagnóstico de hipertensão e/ou diabetes, moradores de Bagé, Rio Grande do Sul, Brasil. Método: estudo transversal de base populacional. Foram entrevistadas 1.593 pessoas com 60 anos ou mais, das quais 947 tinham diagnóstico de hipertensão e/ou diabetes. A prevalência "relações sociais informais" foi distribuída em fraca, moderada e forte. A diferença estatística entre os grupos foi avaliada com teste qui-quadrado e intervalos de confiança de 95\%. Resultados: a prevalência de relações informais fracas foi de $51,0 \%$ sendo estatisticamente significativa entre os idosos com idade superior a 74 anos, menor escolaridade e classificação socioeconômica $\mathrm{C}$ e $\mathrm{D}$, residindo em domicílios multigeracionais, com maior número de pessoas e em áreas cobertas pela Estratégia Saúde da Família. Conclusão: os resultados reforçam a necessidade de desenvolver mecanismos de proteção social a idosos com morbidades crônicas, de modo a minimizar o risco de viver isolado.

Descritores: Apoio social; Idoso; Atenção primária à saúde; Hipertensão; Diabetes mellitus

\footnotetext{
${ }^{1}$ Enfermeira. Mestre em Ciências. Universidade Federal de Pelotas. Pelotas, RS, Brasil. mariangela.soares@gmail.com

${ }^{2}$ Docente no Programa de Pós-graduação em Enfermagem UFPel. Doutor em Epidemiologia. Universidade Federal de Pelotas. Pelotas. RS, Brasil. nunesbp@gmail.com

${ }^{3}$ Enfermeira. Mestre em Ciências. Universidade Federal de Pelotas. Pelotas. Rio Grande do Sul. Brasil. louriele@gmail.com

${ }^{4}$ Enfermeira. Mestre em Enfermagem. Universidade Federal de Pelotas. Pelotas. RS, Brasil. marciane.kessler@hotmail.com

${ }^{5}$ Docente no Programa de Pós-graduação em Enfermagem UFPel. Doutora em Epidemiologia. Universidade Federal de Pelotas. Pelotas. RS, Brasil. aliteia@gmail.com

${ }^{6}$ Docente na Faculdade de Enfermagem UFPel. Doutora em Ciências. Universidade Federal de Pelotas. Pelotas. RS, Brasil. soaresdeisi@gmail.com

${ }^{7}$ Docente no Programa de Pós-graduação em Enfermagem UFPel/ Programa de Pós-graduação em Epidemiologia UFPel. Doutor em Medicina. Universidade Federal de Pelotas. Pelotas, RS, Brasil. luizfacchini@gmail.com

${ }^{8}$ Docente no Programa de Pós-graduação em Enfermagem UFPel. Doutora em Epidemiologia. Universidade Federal de Pelotas. Pelotas, RS, Brasil. elainethume@gmail.com
} 


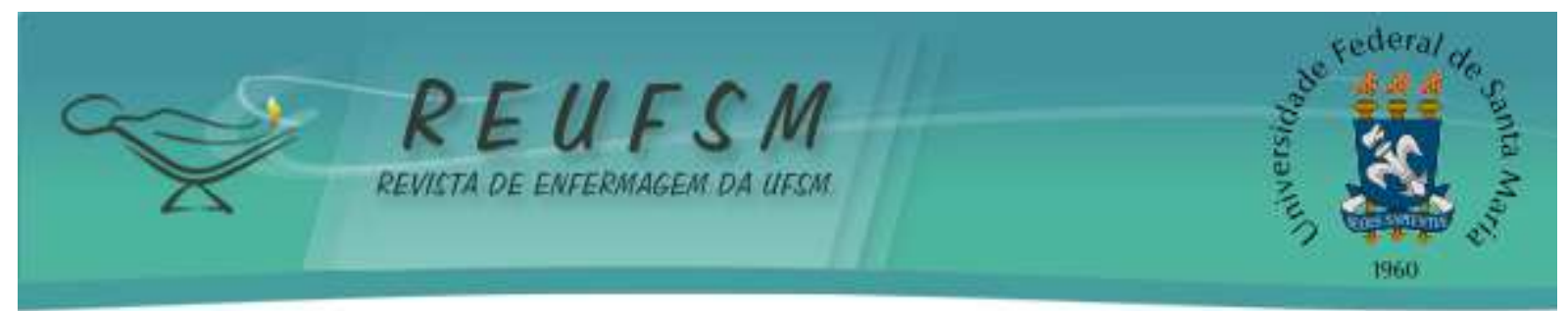

ABSTRACT Aim: to evaluate the informal social relations among the elderly with diagnosis of hypertension and/or diabetes, residing in Bagé, Rio Grande do Sul. Method: crosssectional population-based study. A total of 1,593 people aged 60 and over were interviewed, of whom 947 had a diagnosis of hypertension and/or diabetes. Results: The prevalence of "informal social relations" was distributed in weak, moderate and strong. The statistical difference among the groups was evaluated with chi-square test and confidence intervals of 95\%. Results: the percentage of weak informal relationships was 51.0\%, with higher proportions among the elderly aged over 74 years, lower educational level, $C$ and D socioeconomic classification, residents in multigenerational households, with more people and in areas covered by the "Estratégia Saúde da Familia" health strategy. Conclusion: the results reinforce the need to develop social protection mechanisms for the elderly with chronic morbidity, in order to minimize the risk of living alone.

Descriptors: Social support; Aged; Primary health care; Hypertension; Diabetes mellitus

RESUMEN: Objetivo: evaluar las relaciones sociales informales de los ancianos con diagnóstico de hipertensión y/o diabetes, residentes en Bagé, Rio Grande do Sul. Método: estudio transversal de base poblacional. Fueron entrevistadas 1.593 personas con 60 años o más, de ese total, 947 eran hipertensos y/o diabéticos. La prevalencia "relaciones sociales informales" fue distribuida en débil, moderada y fuerte. La diferencia estadística entre los grupos fue evaluada por medio de prueba qui-cuadrada y intervalos de confianza de $95 \%$. Resultados: El porcentaje de relaciones informales débiles fue de 51,0\%, la cual fue más significativa estadísticamente entre los ancianos mayores de 74 años, con menor escolaridad y clasificación socioeconómica $C$ y $D$, que viven en domicilios multigeracionales, con otras personas, y en áreas asistidas por la Estrategia Salud de la Familia. Conclusión: existe la necesidad de desarrollar mecanismos de protección social a los ancianos con morbilidades crónicas, para minimizar el riesgo de vivir aislado.

Descriptores: Apoyo social; Anciano; Atención primaria de salud; Hipertensión; Diabetes mellitus

\section{INTRODUÇÃO}

No contexto mundial as políticas de promoção do envelhecimento saudável têm destacado a relevância das relações sociais, além das condições físicas e mentais como fatores que otimizam a capacidade funcional e estimulam a saúde. ${ }^{1}$ A atual estrutura demográfica e a modificação do perfil de morbimortalidade, associado à urbanização e industrialização, produzem transformações culturais e sociais, com reflexos diretos na dinâmica e nos vínculos familiares. ${ }^{2}$

O processo de envelhecimento abrange riscos de aumento da vulnerabilidade e de doença, podendo ser agravados por contexto social e rede de apoio social fracas ou inexistentes. ${ }^{3}$ A fragilidade nas relações sociais e/ou a solidão pode ser considerada risco à saúde, ao prejudicar o funcionamento fisiológico, o sono e o bem-estar físico e mental do indivíduo. ${ }^{4}$

A importância das relações sociais é destacada em trabalhos que identificam declínio da saúde física e mental em idosos vivendo em isolamento social, solidão ou com apoio social 


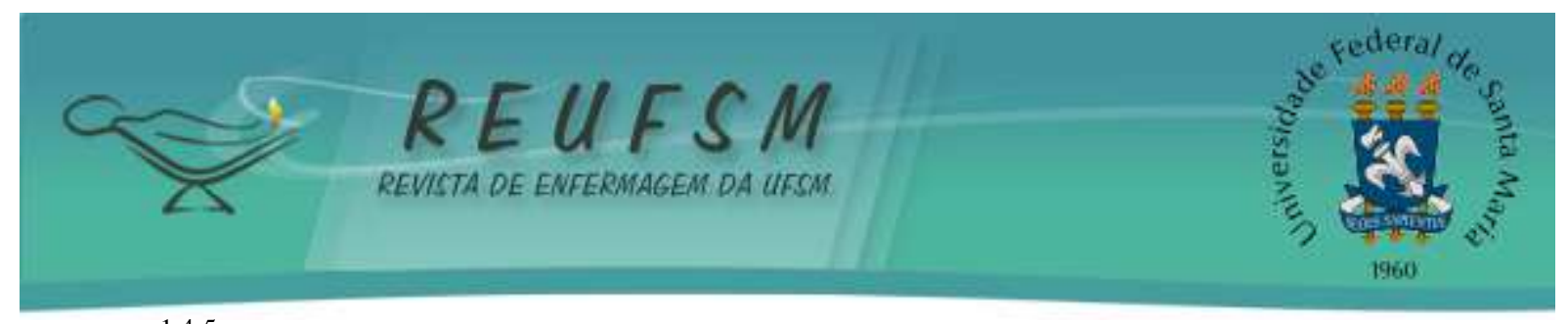

negativo ${ }^{1,4-5}$ e pode ser explicado pelo fato de que a ruptura de laços sociais causa estresse, afetando os sistemas de defesa do organismo tornando os indivíduos suscetíveis a doenças. ${ }^{1}$ Por outro lado, as morbidades e incapacidades podem provocar no indivíduo limitações que acabam por modificar as relações sociais, ${ }^{6-7}$ e afetam o desempenho dos papéis sociais, fragilizando os relacionamentos no trabalho, na família e no círculo de amigos.

No Brasil, estudos epidemiológicos sobre as relações sociais na população idosa, tanto de caráter transversal quanto longitudinal, são recentes. Os achados identificam o efeito protetor das relações sociais sobre a mortalidade, ${ }^{4}$ a morbidade, ${ }^{4}$ a perda funcional, ${ }^{8}$ a perda da função congnitiva ${ }^{9}$ e autopercepção ruim da saúde. ${ }^{10}$

O estudo das relações sociais, ${ }^{11}$ propõe sua divisão em estrutura e função. A estrutura estaria subdividida por relações formais e informais. As relações formais estão relacionadas aos contatos com os prestadores de serviços e, as relações informais, aos vínculos afetivos com membros da família, amigos, vizinhos e colegas de trabalho, podendo estes oferecer ou não auxílio no cuidado ao idoso. ${ }^{11-12}$

O aumento na expectativa de vida e a diminuição no tamanho das famílias alteraram o sistema informal de apoio e, consequentemente, a organização do cuidado informal, reforçando a importância de conhecer o número de pessoas com as quais os indivíduos mantêm contato social, a frequência, a duração, a diversidade, a consistência e a reciprocidade das relações. ${ }^{11,13}$ Estas questões são relevantes, principalmente nas pessoas com doenças crônicas como, por exemplo, hipertensão e diabetes, com prevalências elevadas na população idosa $^{14}$ e tratamentos com necessidade de mudanças comportamentais para as quais os vínculos sociais são fundamentais.

A literatura que estuda as relações informais em indivíduos com doenças crônicas, especialmente hipertensão e diabetes, é escassa e voltada à abordagem qualitativa ou revisão de literatura. ${ }^{15-17}$ Portanto, este estudo responde a seguinte questão: "quais as características das relações sociais informais de idosos com as doenças crônicas hipertensão e diabetes?”, e objetiva avaliar as relações sociais informais dos idosos com diagnóstico de hipertensão e/ou diabetes moradores de Bagé, no Rio Grande do Sul (RS), Brasil. 


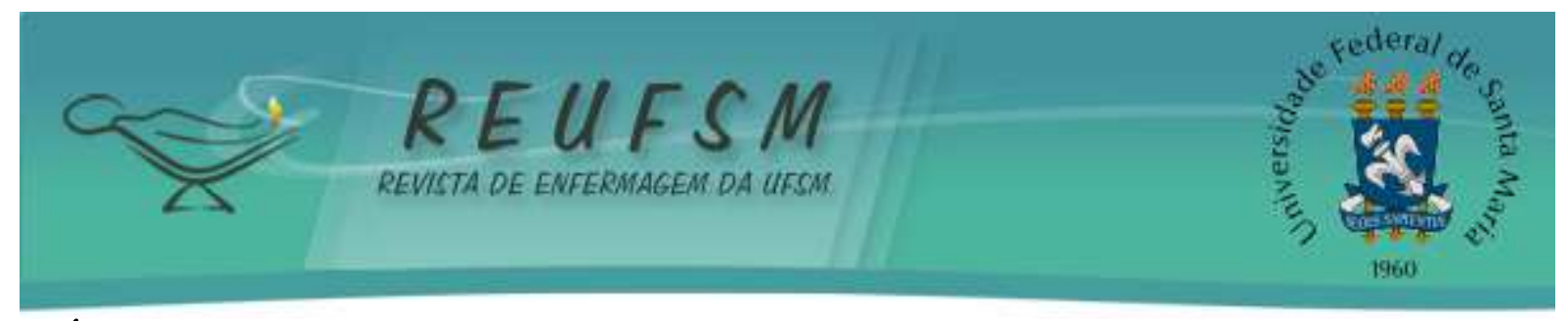

\section{MÉTODO}

Estudo epidemiológico de base populacional, com idosos residentes na zona urbana do município de Bagé, RS, Brasil. No total, foram entrevistados 1.593 idosos com 60 anos ou mais de idade, no período de julho a novembro de 2008.

Foi realizado estudo piloto com idosas residentes em área pertencente a uma instituição de longa permanência, no qual foi permitido visualizar a logística do trabalho de campo e identificar as particularidades da abordagem às pessoas idosas. A amostra foi localizada a partir da área de abrangência de cada uma das Unidades Básicas de Saúde (UBS) e posteriormente dividida em microáreas, com a identificação numérica de cada quadra. O ponto de início da coleta de dados nas quadras foi selecionado aleatoriamente e o domicílio à esquerda foi elegível, com abordagem de um em cada seis domicílios. Todos os moradores com 60 anos ou mais foram convidados a participar do estudo e incluídos mediante leitura e assinatura do Termo de Consentimento Livre e Esclarecido. As entrevistas não realizadas após três tentativas em dias e horários diferentes foram consideradas perdas/recusas. Foram excluídos os indivíduos que, no momento da entrevista, estavam viajando, privados de liberdade por decisão judicial ou residindo em instituição de longa permanência.

A coleta dos dados foi realizada por meio de questionário estruturado com questões pré-codificadas e aplicado no domicílio por 15 entrevistadores, coordenados por três supervisores. Existiam 15 unidades de ESF, cobrindo $51 \%$ da população e 5 UBS com modelo de atenção tradicional, responsáveis pelo atendimento dos demais habitantes.

O desfecho 'relações sociais informais' foi construído a partir de um instrumento adaptado $^{11,13}$ utilizando cinco questões, com possibilidade de resposta dicotômica (sim, não): “A sua família lhe visitou?”; “O $\operatorname{Sr}($ a) foi visitar a sua família?”; "Foi visitar seus amigos?”; "Seus amigos lhe visitaram?”; “Teve contato telefônico ou por carta com seus parentes ou amigos?". Em caso de resposta positiva era questionado o número de vezes que teve contato com amigos e familiares nos quinze dias anteriores à entrevista. Presumiu-se que a utilização do recordatório da quinzena precedente à coleta de dados facilitaria ao idoso lembrar os acontecimentos recentes e também a investigação das relações sociais cotidianas, com maior probabilidade de indicar uma semana típica do indivíduo.

As respostas foram pontuadas da seguinte forma: respostas negativas = um ponto; um a dois contatos $=$ dois pontos; três a seis contatos $=$ três pontos e sete contatos ou mais $=$ 


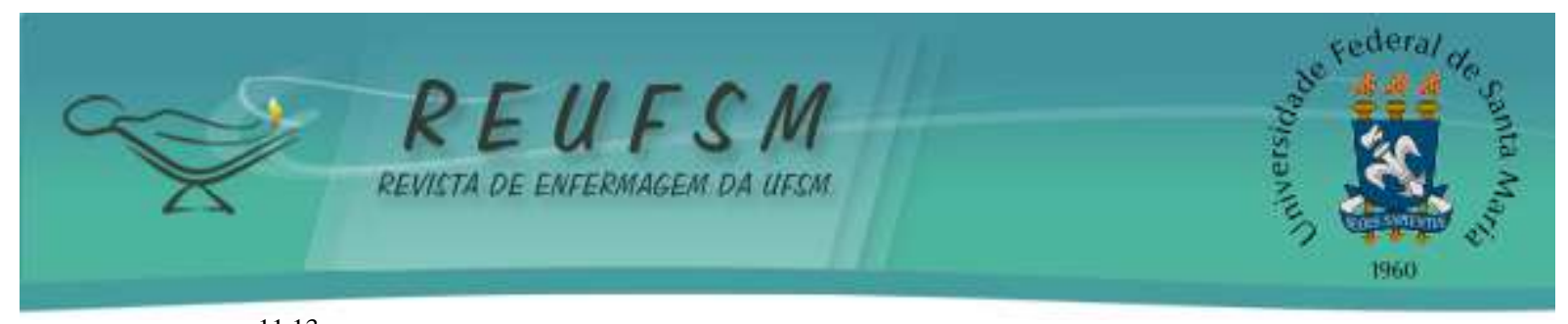

quatro pontos. ${ }^{11,13}$ Foi construído um escore e as relações informais foram classificadas em fracas (cinco a nove pontos), moderadas (dez a 14 pontos) ou fortes (15 pontos ou mais).

Os idosos com diagnóstico de hipertensão e/ou diabetes foram identificados por meio das seguintes perguntas com opção de resposta dicotômica (sim; não): "Algum médico disse que o(a) Sr.(a) tem pressão alta?”; "Algum médico disse que o(a) Sr.(a) tem diabetes ou açúcar alto no sangue?".

O contexto demográfico e socioeconômico foi analisado por meio das seguintes variáveis independentes: sexo (masculino; feminino), idade (60 a 74; 75 anos ou mais), cor da pele autorreferida (branca; preta; amarela, parda ou indígena), situação conjugal (casado ou com companheiro; viúvo; solteiro ou separado), número de moradores no domicílio (mora sozinho, duas pessoas; três pessoas; quatro ou mais), domicílio multigeracional (não; sim), classificação econômica da Associação Brasileira de Empresas de Pesquisas - ABEP (A/B; C; D/E), anos de estudo - em anos completos (nenhum; um a sete; e oito ou mais), aposentado (não; sim) e Modelo de Atenção da UBS (Tradicional e Estratégia Saúde da Família).

Para a análise dos dados foi realizado cálculo de distribuição proporcional com apresentação dos intervalos de confiança (IC) de $95 \%$ e a diferença estatística entre os grupos por meio do teste de Qui-quadrado para heterogeneidade (p-valor). A análise foi realizada no programa estatístico Stata versão 12.0 (Stata Corporation, College Station, EUA).

Os preceitos éticos foram respeitados e o projeto foi aprovado no Comitê de Ética em Pesquisa da Faculdade de Medicina da UFPel, com registro $n^{\circ} 406.00 .036$ (ofício $n^{\text {o. }}$ 015/08 25 de agosto de 2008). A Fundação de Amparo à Pesquisa do Rio Grande do Sul FAPERGS, apoiou a realização deste estudo.

\section{RESULTADOS}

Do total entrevistados $(n=1.593)$ a proporção de idosos com hipertensão e/ou diabetes foi de 59,4\% ( $\mathrm{n}=947)$. As mulheres representaram $63 \%$ da amostra total e praticamente um terço $(31,2 \%)$ tinha 75 anos ou mais de idade. A cor da pele branca foi referida por 78,6\% dos idosos. Metade era casada ou com companheiro (51,2\%). A proporção de idosos que residiam sozinhos foi de $17,5 \%$ e a proporção de domicílios com quatro ou mais moradores foi de 27,2\%. Quanto à classificação econômica, 34,0\% pertencia à categoria 


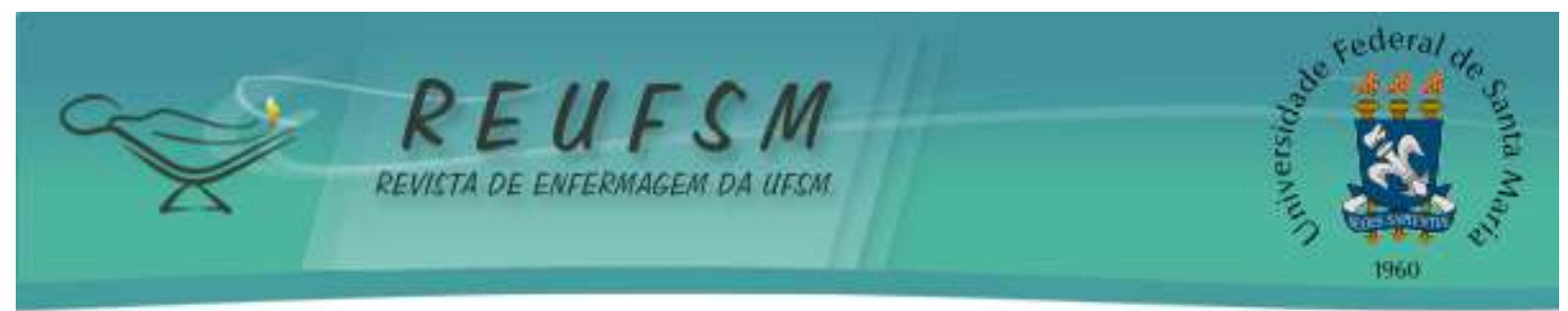

D/E. Quase um quarto da amostra não possuía nenhum ano de estudo completo $(23,7 \%)$. Aproximadamente três quartos $(71,7 \%)$ estavam aposentados (Tabela 1$)$.

Tabela 1 - Distribuição proporcional das características demográficas e socioeconômicas no total da amostra e entre a população idosa portadora de hipertensão e/ou diabetes. Bagé, RS, 2008

\begin{tabular}{|c|c|c|c|c|}
\hline \multirow[t]{2}{*}{ Variáveis } & \multicolumn{2}{|c|}{ Amostra Geral } & \multicolumn{2}{|c|}{$\begin{array}{c}\text { Portadores de } \\
\text { Hipertensão e/ou } \\
\text { Diabetes }\end{array}$} \\
\hline & $\mathrm{n}$ & $\%$ & $\mathrm{~N}$ & $\%$ \\
\hline \multicolumn{5}{|l|}{ Sexo } \\
\hline Masculino & 593 & 37,2 & 297 & 31,4 \\
\hline Feminino & 1.000 & 62,8 & 650 & 68,6 \\
\hline \multicolumn{5}{|l|}{ Idade (em anos completos) } \\
\hline 60 a 74 & 1.096 & 68,8 & 656 & 69,3 \\
\hline 75 ou mais & 497 & 31,2 & 291 & 30,7 \\
\hline \multicolumn{5}{|l|}{ Cor da pele (autorreferida) } \\
\hline Branca & 1.252 & 78,6 & 739 & 78,0 \\
\hline Preta & 139 & 8,7 & 84 & 8,9 \\
\hline Parda/amarela/indígena & 202 & 12,7 & 124 & 13,1 \\
\hline \multicolumn{5}{|l|}{ Situação Conjugal } \\
\hline Casado(a) ou com companheiro(a) & 816 & 51,2 & 477 & 50,4 \\
\hline Solteiro(a) ou Separado(a) & 238 & 15,0 & 131 & 13,9 \\
\hline Viúvo(a) & 538 & 33,8 & 338 & 35,7 \\
\hline \multicolumn{5}{|l|}{ Número de moradores no domicílio } \\
\hline Mora sozinho & 279 & 17,5 & 163 & 17,3 \\
\hline Dois & 538 & 33,8 & 302 & 32,0 \\
\hline Três & 342 & 21,5 & 201 & 21,3 \\
\hline Quatro ou mais & 432 & 27,2 & 279 & 29,4 \\
\hline \multicolumn{5}{|l|}{ Domicílio multigeracional } \\
\hline Não & 759 & 47,6 & 420 & 44,4 \\
\hline Sim & 834 & 52,4 & 527 & 55,6 \\
\hline \multicolumn{5}{|l|}{ Classificação econômica (ABEP) } \\
\hline $\mathrm{A} / \mathrm{B}$ & 429 & 27,1 & 244 & 26,0 \\
\hline $\mathrm{C}$ & 615 & 38,9 & 381 & 40,6 \\
\hline $\mathrm{D} / \mathrm{E}$ & 537 & 34,0 & 314 & 33,4 \\
\hline \multicolumn{5}{|l|}{ Anos de estudo (em anos completos) } \\
\hline Nenhum & 372 & 23,7 & 228 & 24,4 \\
\hline 1 a 7 & 858 & 54,5 & 517 & 55,4 \\
\hline$\geq 8$ & 342 & 21,8 & 189 & 20,2 \\
\hline \multicolumn{5}{|l|}{ Aposentadoria } \\
\hline Não & 451 & 28,3 & 277 & 29,3 \\
\hline Sim & 1.142 & 71,7 & 670 & 70,7 \\
\hline \multicolumn{5}{|l|}{ Modelo de Atenção da UBS } \\
\hline Tradicional & 741 & 46,5 & 441 & 46,6 \\
\hline Estratégia Saúde da Família & 852 & 53,5 & 506 & 53,4 \\
\hline Total & 1.593 & 100,0 & 947 & 100,00 \\
\hline
\end{tabular}

Nos últimos quinze dias, $77,9 \%$ dos idosos recebeu visita dos familiares e entre aqueles com hipertensão e/ou diabetes esta proporção foi de $80,5 \%$. A proporção de visitas feitas pelos idosos a membros da família foi menor, metade dos idosos havia saído de casa 


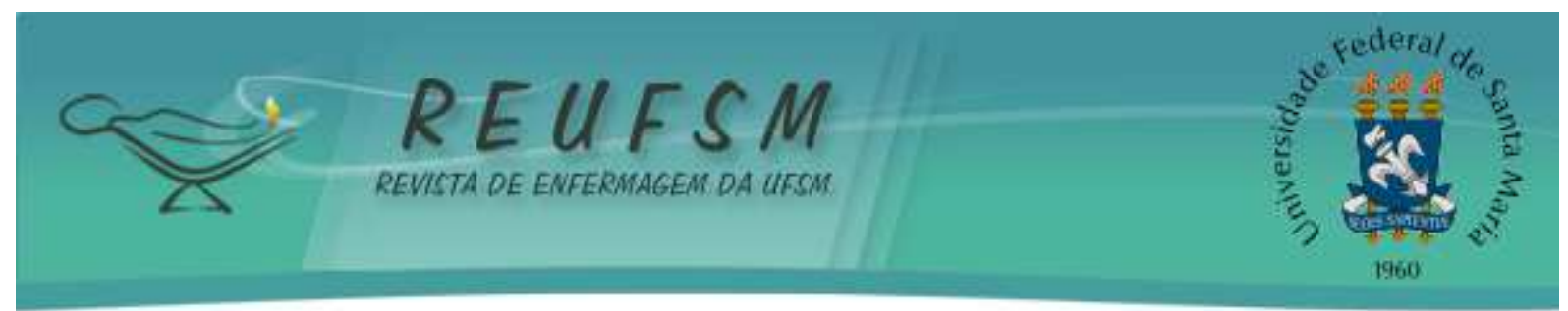

para visitar a família $(52,7 \%$ da população total e $51,7 \%$ dos idosos com hipertensão e/ou diabetes). Cerca de $67,0 \%$ recebeu visita dos amigos, independente de ser ou não doente crônico. Neste mesmo período, $44,4 \%$ dos idosos visitou os amigos e esta proporção foi de $43,3 \%$ entre aqueles com hipertensão e/ou diabetes. O contato telefônico ou por carta com parentes ou amigos foi referido por 70,0\% dos idosos.

Relações informais fracas foram observadas em praticamente metade da amostra, sendo $52,5 \%$ ( $\left.\mathrm{IC}_{95 \%} 50,0 \%-55,0 \%\right)$ na população total e 50,9\% ( $\left.\mathrm{IC}_{95 \%} 47,7 \%-54,1 \%\right)$ nas pessoas com hipertensão e diabetes. A proporção de relações sociais informais fortes foi de aproximadamente 5,0\%, sem diferença entre os grupos (Figura 1).

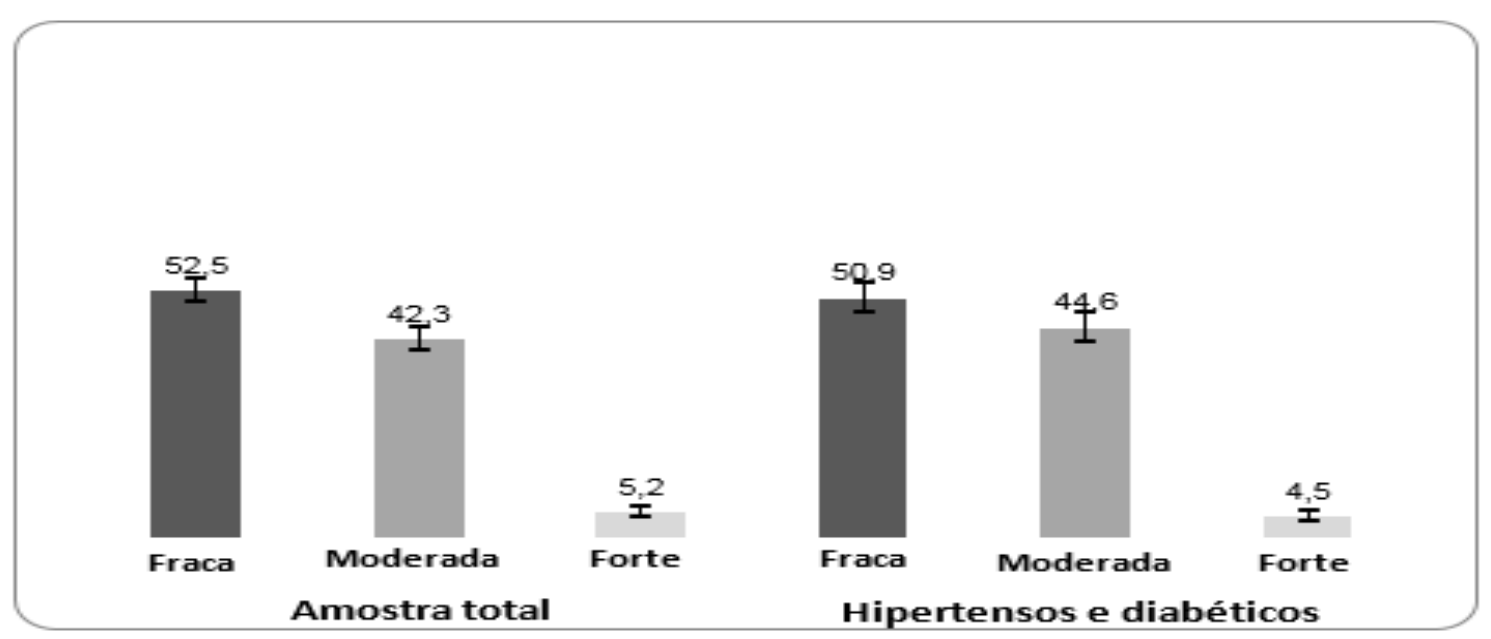

Figura 1 - Distribuição proporcional do escore das relações informais na amostra total e entre os portadores de hipertensão e/ou diabetes. Bagé, RS, 2008

A probabilidade de ter relações informais fracas aumentou cerca de $21,0 \%$ entre os idosos hipertensos e/ou diabéticos com 75 anos ou mais, comparados àqueles mais jovens e entre idosos residentes em domicílios multigeracionais. Houve uma tendência à piora das relações informais conforme o aumento do número de moradores no domicílio. Nos indivíduos que residiam com quatro ou mais pessoas, a probabilidade de enfraquecimento das relações informais era 53,0\% maior quando comparada aos indivíduos que moravam sozinhos. Nos idosos pertencentes à classificação $\mathrm{C}$ e D/E, a probabilidade de apresentar relação fraca foi maior se comparados àqueles com classificação econômica $\mathrm{A}(43,0 \%$ e $77,0 \%$, respectivamente). A escolaridade apresentou-se como fator de proteção para as relações sociais fracas, ou seja, a probabilidade dos indivíduos com oito anos ou mais de estudo ter uma relação fraca era menor quando comparada àqueles que não estudaram. Idosos 


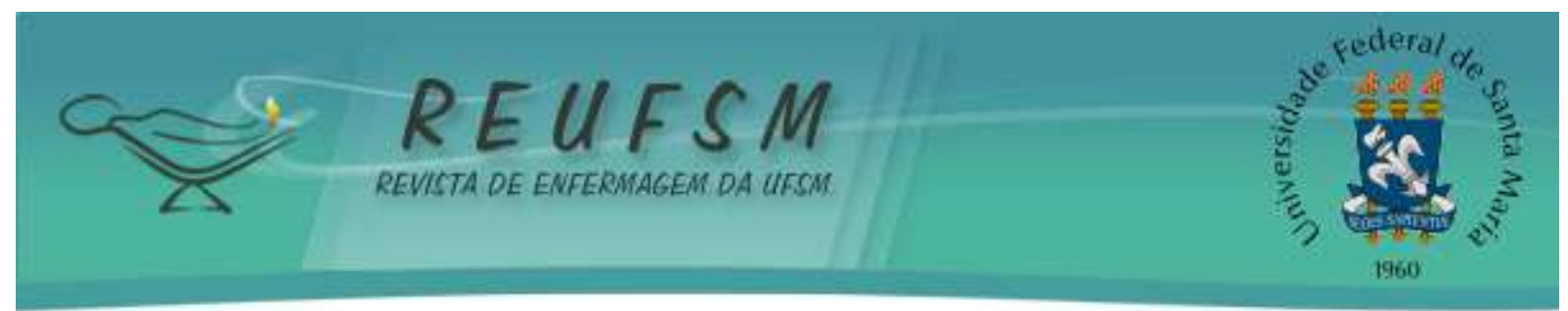

domiciliados em áreas cobertas pela Estratégia Saúde da Família (ESF) apresentavam 25\% mais probabilidade de apresentarem relações informais fracas comparados aos residentes em áreas com unidades de modelo de atenção tradicional (Tabela 2).

As maiores proporções de relações informais fracas foram observadas entre os homens $(53,7 \%)$, naqueles que autorreferiram cor da pele preta $(59,5 \%)$, nos solteiros e viúvos e/ou nos não aposentados (53,5\%), entretanto estas diferenças não foram estatisticamente significativas (Tabela 2).

Tabela 2 - Prevalência de relações informais fracas entre idosos com hipertensão e/ou diabetes e associação com as variáveis independentes $(n=482)$. Bagé, RS, 2008

\begin{tabular}{|c|c|c|}
\hline \multirow[t]{2}{*}{ Variáveis } & \multicolumn{2}{|c|}{ Relações Informais Fracas } \\
\hline & $\mathrm{N}$ & $\%\left(\mathrm{IC}_{95 \%}\right)$ e valor-p* \\
\hline Sexo & & $\mathrm{p}=0,291$ \\
\hline Masculino & 159 & $53,6(47,8 ; 59,3)$ \\
\hline Feminino & 323 & $49,7(45,8 ; 53,6)$ \\
\hline Idade (em anos completos) & & $\mathrm{p}=0,005$ \\
\hline 60 a 74 & 314 & $47,8(43,9 ; 51,6)$ \\
\hline 75 ou mais & 169 & $58,0(52,3 ; 63,7)$ \\
\hline Cor da pele (autorreferida) & & $\mathrm{p}=0,145$ \\
\hline Branca & 364 & $49,3(45,7 ; 53,0)$ \\
\hline Preta & 50 & $59,5(48,8 ; 70,2)$ \\
\hline Parda/Amarela/Indígena & 68 & $54,5(45,6 ; 63,4)$ \\
\hline Situação Conjugal & & $\mathrm{p}=0,770$ \\
\hline Casado(a) ou com companheiro(a) & 237 & $49,7(45,1 ; 54,2)$ \\
\hline Solteiro(a) ou Separado(a) & 68 & $51,9(43,2 ; 60,6)$ \\
\hline Viúvo(a) & 176 & $52,1(46,7 ; 57,5)$ \\
\hline Número de moradores na casa & & $\mathrm{p}<0,001$ \\
\hline Mora sozinho & 67 & $41,4(33,7 ; 49,0)$ \\
\hline Dois & 137 & $45,2(39,5 ; 50,8)$ \\
\hline Três & 100 & $49,8(42,7 ; 56,8)$ \\
\hline Quatro ou mais & 177 & $63,5(57,8 ; 69,2)$ \\
\hline Domicílio multigeracional & & $\mathrm{p}=0,004$ \\
\hline Não & 191 & $45,5(40,7 ; 50,4)$ \\
\hline Sim & 291 & $55,2(51,0 ; 59,5)$ \\
\hline Classificação econômica (ABEP) & & $\mathrm{p}<0,001$ \\
\hline $\mathrm{A} / \mathrm{B}$ & 86 & $35,3(29,2 ; 41,4)$ \\
\hline $\mathrm{C}$ & 192 & $50,5(45,5 ; 55,6)$ \\
\hline $\mathrm{D} / \mathrm{E}$ & 197 & $62,6(57,2 ; 68,0)$ \\
\hline Escolaridade (anos) & & $\mathrm{p}<0,001$ \\
\hline Nenhum & 144 & $63,3(56,9 ; 69,6)$ \\
\hline
\end{tabular}




\begin{tabular}{|c|c|c|}
\hline${ }_{\text {REVIS }}$ & & $\frac{z^{2}}{2}$ federal $\%$ \\
\hline Um a sete & 266 & $51,5(47,1 ; 55,8)$ \\
\hline Oito ou mais & 64 & $33,9(27,1 ; 40,7)$ \\
\hline Aposentadoria & & $\mathrm{p}=0,316$ \\
\hline Não & 148 & $53,5(47,5 ; 59,4)$ \\
\hline Sim & 334 & $49,9(46,0 ; 53,7)$ \\
\hline Modelo de Atenção da UBS & & $\mathrm{p}<0,001$ \\
\hline Tradicional & 198 & $44,9(40,2 ; 49,5)$ \\
\hline Estratégia Saúde da Família & 284 & $56,2(51,8 ; 60,6)$ \\
\hline
\end{tabular}

\section{DISCUSSÃO}

Neste estudo, o percentual de relações informais fracas entre os idosos com hipertensão e/ou diabetes foi de 51,0\% ( $\mathrm{IC}_{95 \%} 47,7 \%-54,1 \%$ ), com proporções maiores entre os idosos com idade superior a 75 anos, menos anos de estudo, moradores de domicílios multigeracionais e com maior número de pessoas, classificação socioeconômica desfavorecida e moradores nas áreas cobertas pela ESF.

O papel protetor de uma rede social positiva e estável no manejo de doenças é importante, principalmente pelo potencial de busca no acesso à utilização de serviços de saúde, pela manutenção da saúde mental e, consequentemente, pela capacidade resolutiva dos problemas de saúde, prevenindo complicações. ${ }^{5,7}$ Contudo, salienta-se que as condições de saúde e a presença de doenças crônicas, afetam a interação social entre os indivíduos, podendo reduzir o tamanho, a qualidade e dificultar sua função. ${ }^{7}$

Estudos apontaram que o apoio das relações informais, prestado tanto pelos familiares e/ou pelos vizinhos e amigos influencia no cuidado recebido pelo indivíduo hipertenso, ${ }^{15-16}$ podendo favorecer a adesão ao tratamento a partir de mudanças no estilo de vida e, consequentemente, melhorar a qualidade de vida. ${ }^{16}$ Em estudo de revisão, ${ }^{17}$ autores sugerem que níveis mais altos de apoio social estão associados a melhores resultados clínicos, estabilidade mental e a adaptação às atividades benéficas de estilo de vida em diabéticos. Pesquisa realizada com idosos em São Paulo identificou que a família é o principal suporte social deste grupo etário, ${ }^{18}$ o que é fundamental para o envelhecimento saudável.

$\mathrm{O}$ aumento da prevalência de relações informais fracas em idosos mais velhos corrobora com a literatura ${ }^{7}$ que destaca a diminuição do envolvimento social com o avançar da idade. O incremento da idade em idosos está associado a incapacidade funcional, ${ }^{19}$ que 


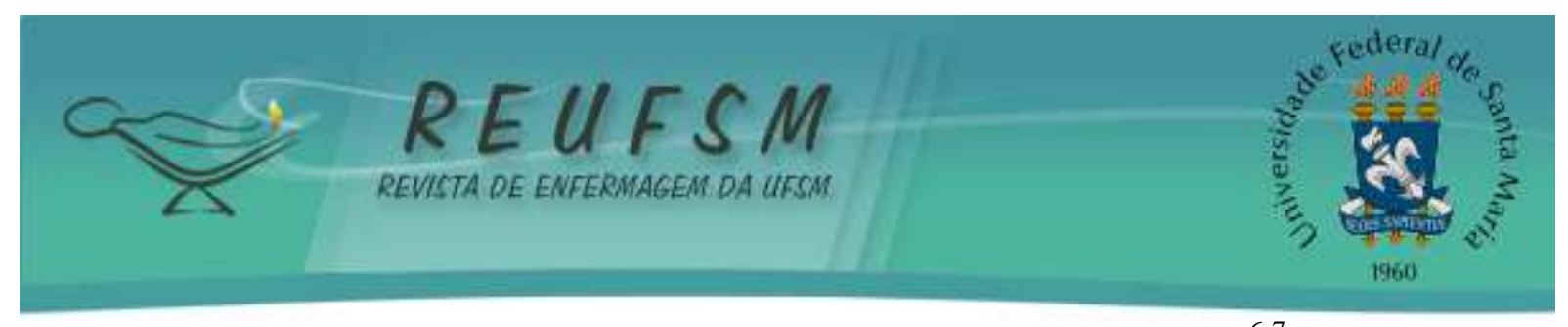

provoca no indivíduo limitações que podem modificar as relações sociais. ${ }^{6-7}$ Além disso, em idade avançadas as perdas se tornam mais frequentes, como do cônjuge, de amigos e de outros familiares, o que pode fragilizar as relações informais do idoso.

$\mathrm{O}$ aumento das relações informais fracas em idosos com baixa escolaridade e de baixa renda demonstra os efeitos dos fatores socioeconômicos na vida social e, consequentemente, na saúde e na qualidade de vida dos indivíduos, ${ }^{20}$ reforçando a necessidade de melhorar e ampliar as redes de apoio entre os grupos socioeconomicamente desfavorecidos. Estudos realizados com idosos em localidades brasileiras com distintos níveis de desenvolvimento socioeconômico, destacaram que a população de maior renda, ${ }^{7}$ com melhor nível de escolaridade e os residentes nas localidades economicamente mais desenvolvidas ${ }^{21}$ apresentaram maior envolvimento social.

A presença de maior percentual de relações informais fracas entre os idosos com hipertensão e/ou diabetes moradores nas áreas cobertas pela ESF pode estar relacionado à prerrogativa da implantação deste modelo de atenção à saúde em áreas de maior vulnerabilidade socioeconômica e em áreas periféricas, sendo que, quando o estudo foi realizado em Bagé em 2008, a cobertura era de 51,0\% abrangendo as regiões mais desfavorecidas no contexto socioeconômico. ${ }^{22}$ Vale ressaltar que a população idosa residente na periferia frequentemente vivencia maior dificuldade de acesso ao transporte, o que pode impactar negativamente as relações informais.

Neste estudo em Bagé, as variáveis de contexto mostraram-se importantes marcadores de fragilização das relações informais. Ou seja, indivíduos que viviam em áreas cobertas pela ESF poderiam ter suas relações interpessoais incentivadas pela equipe de saúde, pois estas teriam o potencial de estimular e apoiar a ação de redes de suporte, a partir do enfrentamento de problemas relacionados à saúde. ${ }^{23}$

Compreende-se que o tratamento e a prevenção contra os agravos clínicos provocados pela hipertensão ou diabetes devem incluir mudanças comportamentais com reflexos diretos nos hábitos individuais e familiares. ${ }^{15}$ Sendo assim, entende-se que a ESF, por se tratar de uma política pública com ênfase na abordagem familiar e comunitária, pode promover ações articuladas e corresponsabilidades entre família, rede de apoio social e serviços de saúde. ${ }^{22}$

Os profissionais de saúde podem contribuir com o fortalecimento dos vínculos sociais ativos de seus usuários ao estimular o engajamento e apoio social, na melhoria da qualidade 


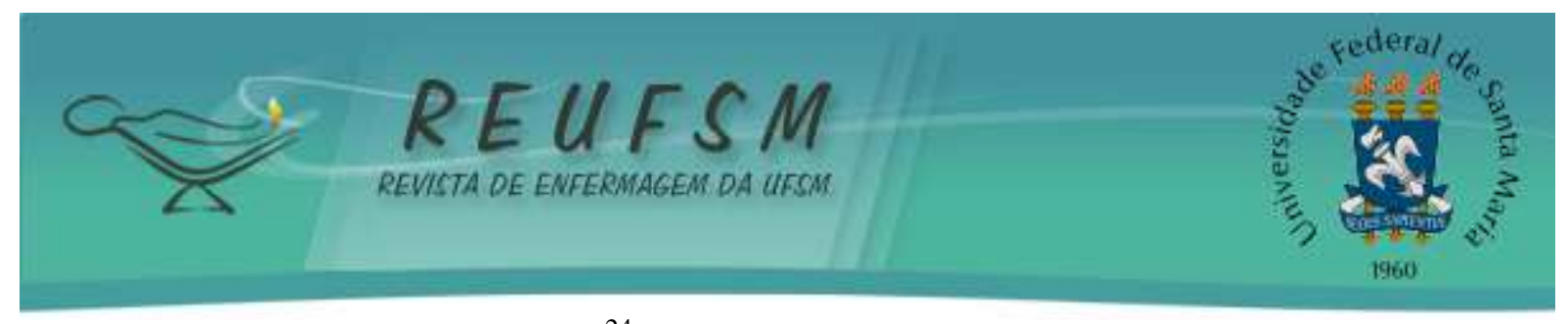

de vida e na sobrevida dos mesmos. ${ }^{24}$ Por se traduzir no contato dos idosos com seus parentes, amigos e vizinhos, a investigação das relações informais poderá ser utilizada como um marcador para o isolamento social e para a baixa adesão ao tratamento de doenças crônicas. Neste sentido, é necessário identificar os idosos com doenças crônicas em risco, e assegurar a sua conexão social com familiares e comunidades locais. ${ }^{5}$

Estes dados reforçam o papel dos aspectos sociais dos indivíduos sobre a sua saúde física e mental, e a necessidade de inseri-los em programas voltados para a promoção do envelhecimento saudável. Além de estudos voltados à população socialmente vulnerável, para conhecer a sua realidade e reorientar a atenção integral à saúde ao idoso. ${ }^{10}$

O município de Bagé possui, atualmente, uma boa organização política para o atendimento às necessidades do idoso. Além da Secretaria de Políticas Públicas para a Pessoa Idosa e do Conselho Municipal dos Direitos do Idoso existe o Centro do Idoso, fundado em 2008, tem uma sede ampla que abriga várias atividades de inclusão social para este grupo. Os grupos exercem um importante papel na vida dos idosos, pois nestes momentos são estimulados hábitos para uma vida social sadia, desenvolvimento da sua cultura e momentos de lazer, o que melhora sua autoestima e sua aceitação na sociedade e auxilia a enfrentar as demandas que afetam o seu bem-estar. ${ }^{25}$ Por isto, é importante que os profissionais da saúde estabeleçam parcerias e incentivem a vinculação de seus usuários aos serviços de apoio social.

\section{CONCLUSÃO}

Ao avaliar as relações informais entre idosos com hipertensão e/ou diabetes observouse que as relações classificadas como fracas apresentaram proporções estatisticamente significativas entre os idosos mais longevos, com menor escolaridade, moradores em domicílios com maior número de pessoas e multigeracionas, classificação socioeconômica desfavorecida e moradores nas áreas cobertas pela ESF, portanto com um possível efeito negativo na qualidade de vida do idoso. Neste contexto, a atenção primária possui um importante papel no reconhecimento das necessidades em saúde do idoso e incentivo ao fortalecimento dos vínculos e redes sociais.

Neste estudo, o instrumento utilizado limitou-se a estrutura e função das relações informais, não considerando as características culturais e as relações de proximidade, sendo fundamental explorar as questões que permeiam essa necessidade, por meio do 


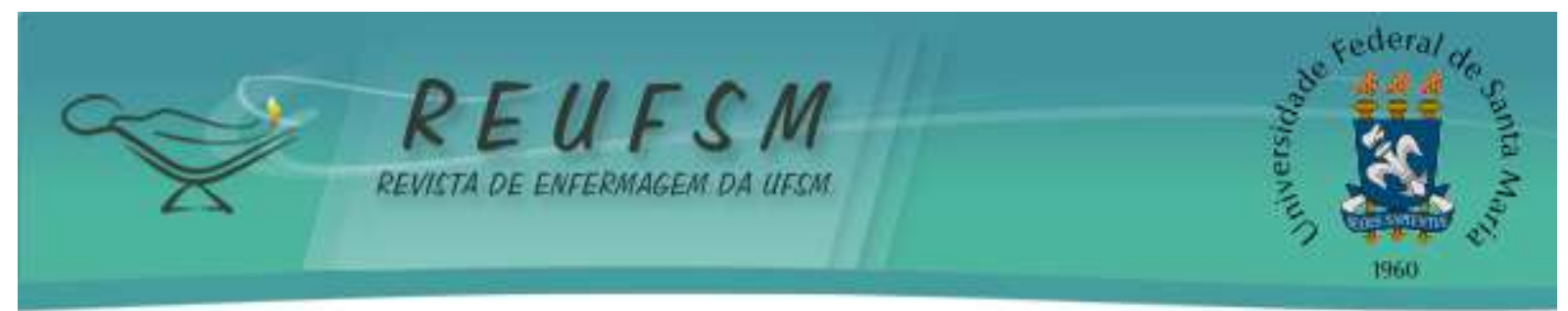

desenvolvimento de pesquisas qualitativas com foco na caracterização proximal dos vínculos, das redes, da qualidade dos contatos realizados e não somente a quantidade de vezes que o contato foi estabelecido. Ainda, indica-se ampliar a análise da função das relações sociais, entre elas apoio emocional, apoio instrumental, apoio de informações, conflitos e integração social.

\section{REFERÊNCIAS}

1. Stenner P, McFarquhar T, Bowling A. Older people and 'active ageing': subjective aspects of ageing actively. J Health Psychol [Internet]. 2012 [acesso em 2017 set 06];16(3):467-77. Disponível em: http://oro.open.ac.uk/30122/2/9BEE017F.pdf.

2. Camarano AA, organizador. Novo regime demográfico: uma nova relação entre população e desenvolvimento? Rio de Janeiro: Ipea; 2014. 658p.

3. Maia CML, Castro FV, Fonseca AMG, Fernandéz MIR. Redes de apoio social e de suporte social e envelhecimento ativo. INFAD Psicol Infanc Adolesc [Internet]. 2016 [acesso em 2017 set 06];1(1):293-303. Disponível em: http://www.infad.eu/RevistaINFAD/OJS/index.php/IJODAEP/article/view/279/182.

4. Cacioppo JT, Cacioppo S. Social relationships and health: the toxic effects of perceived social isolation. Soc Personal Psychol Compass [Internet]. 2014 [acesso em 2017 jun 20];8(2):58-72. Disponível em: https://www.ncbi.nlm.nih.gov/pubmed/24839458.

5. Ahn SN, Kim S, Zhang H. Changes in depressive symptoms among older adults with multiple chronic conditions: role of positive and negative social support. Int J Environ Res Public Health [Internet]. 2017 [acesso em 2017 jun 20];14(1):1-11. Disponível em: https://www.ncbi.nlm.nih.gov/pmc/articles/PMC5295267/.

6. Rosso AL, Taylor JA, Tabb LP, Michael YL. Mobility, disability, and social engagement in older adults. J Aging Health [Internet]. 2013 [acesso em 2017 jun 20];25(4):617-37. Disponível

em: https://www.ncbi.nlm.nih.gov/pmc/articles/PMC3683993/pdf/nihms471291.pdf.

7. Pinto JM, Neri AL. Doenças crônicas, capacidade funcional, envolvimento social e satisfação em idosos comunitários: Estudo Fibra. Ciênc Saúde Colet [Internet]. 2013 [acesso em 2017 jun 20];18(12):3449-60. Disponível em: http://www.scielo.br/pdf/csc/v18n12/a02v18n12.pdf.

8. Amaral FLJS, Guerra RO, Nascimento AFF, Maciel ÁCC. Apoio social e síndrome da fragilidade em idosos residentes na comunidade. Ciênc Saúde Colet [Internet]. 2013 [acesso em 2017 set 06];18(6):1835-46. Disponível em: http://www.scielo.br/pdf/csc/v18n6/34.pdf.

9. Brito TRP, Pavarini SCI. Relação entre apoio social e capacidade funcional de idosos com alterações cognitivas. Rev Latinoam Enferm [Internet]. 2012 [acesso em 2017 set 06];20(4):677-84. Disponível em: http://www.scielo.br/pdf/rlae/v20n4/pt_07.pdf.

10. Nunes APN, Barreto SM, Gonçalves LG. Relações sociais e autopercepção da saúde: Projeto Envelhecimento e Saúde. Rev Bras Epidemiol [Internet]. 2012 [acesso em 2017 jun 20];15(2):415-28. Disponível em: http://www.scielo.br/pdf/rbepid/v15n2/19.pdf. 


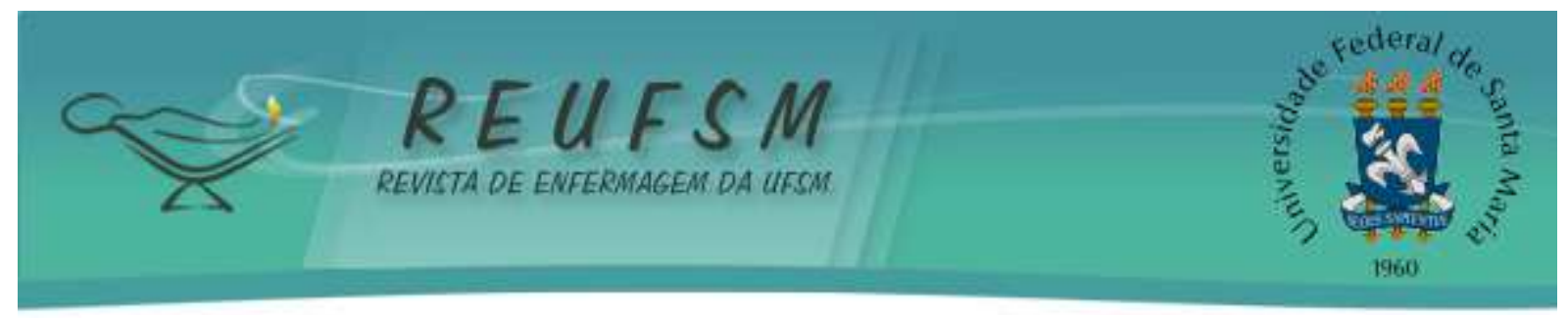

11. Due P, Holstein B, Lund R, Modvig J, Avlund K. Social relations: network, support and relational strain. Soc Sci Med [Internet]. 1999 [acesso em 2017 jun 20];48(5):661-73. Disponível em: https://www.ncbi.nlm.nih.gov/pubmed/10080366.

12. Anjos KF, Boery RNSO, Pereira R, Pedreira LC, Vilela ABA, Santos VC, et al. Associação entre apoio social e qualidade de vida de cuidadores familiares de idosos dependentes. Ciênc Saúde Colet [Internet]. 2015 [acesso em 2017 jun 20];20(5):1321-30. Disponível em: http://www.scielo.br/pdf/csc/v20n5/pt_1413-8123-csc-20-05-01321.pdf.

13. Rosa TEC. Redes de apoio social. In: Litvoc J, Brito FC, editores. Envelhecimento: prevenção e promoção da saúde. São Paulo (SP): Atheneu; 2004. p. 203-18.

14. Nunes BP, Thumé E, Facchini LA. Multimorbidity in older adults: magnitude and challenges for the Brazilian health system. BMC public health [Internet]. 2015 [acesso em 2017 jun 20];15(1172):1-11. Disponível em: https://www.ncbi.nlm.nih.gov/pmc/articles/PMC4658761/.

15. Trad LAB, Tavares JSC, Soares CS, Ripardo RC. Itinerários terapêuticos face à hipertensão arterial em famílias de classe popular. Cad Saúde Pública [Internet]. 2010 [acesso em 2017 jun 20];26(4):797-806. Disponível em: http://www.scielo.br/pdf/csp/v26n4/21.pdf.

16. Faquinello P, Marcon SS. Amigos e vizinhos: uma rede social ativa para adultos e idosos hipertensos. Rev Esc Enferm USP [Internet]. 2011 [acesso em 2017 jun 20];45(6):1345-52. Disponível em: http://www.scielo.br/pdf/reeusp/v45n6/v45n6a10.pdf.

17. Strom JL, Egede LE. The impact of social support on outcomes in adult patients with type 2 diabetes: a systematic review. Curr Diab Rep [Internet]. 2012 [acesso em 2017 jun 20];12(6):769-81. Disponível em: https://www.ncbi.nlm.nih.gov/pmc/articles/PMC3490012/.

18. Domingues MA, Ordonez TN, Lima-Silva TB, Torres MJ, Barros TC, Florindo AA. Redes de relações sociais dos idosos residentes em Ermelino Matarazzo, São Paulo: um estudo epidemiológico. Rev Bras Geriatr Gerontol [Internet]. 2013 [acesso em 2017 jun 20];16(1):49-59. Disponível em: http://www.scielo.br/pdf/rbgg/v16n1/a06v16n1.pdf.

19. Nunes JD, Saes MO, Nunes BP, Siqueira FCV, Soares DC, Fassa MEG, et al. Functional disability indicators and associated factors in the elderly: a population-based study in Bagé, Rio Grande do Sul, Brazil. Epidemiol Serv Saúde [Internet]. 2017 [acesso em 2017 jun 20];26(2):295-304. Disponível em: http://www.scielo.br/pdf/ress/v26n2/en_2237-9622-ress26-02-00295.pdf.

20. Andrade JMO, Rios LR, Teixeira LS, Vieira FS, Mendes DC, Vieira MA, et al. Influência de fatores socioeconômicos na qualidade de vida de idosos hipertensos. Ciênc Saúde Colet [Internet]. 2014 [acesso em 2017 jun 20];19(8):3497-504. Disponível em: http://www.scielo.br/pdf/csc/v19n8/1413-8123-csc-19-08-03497.pdf.

21. Neri AL, Vieira LAM. Envolvimento social e suporte social percebido na velhice. Rev Bras Geriatr Gerontol [Internet]. 2013 [acesso em 2017 jun 20];16(3):419-32. Disponível em: http://www.scielo.br/pdf/rbgg/v16n3/v16n3a02.pdf.

22. Thumé E, Facchini LA, Wyshak G, Campbell P. The utilization of home care by the elderly in Brazil's primary health care system. Am J Public Health. [Internet]. 2011 [acesso 


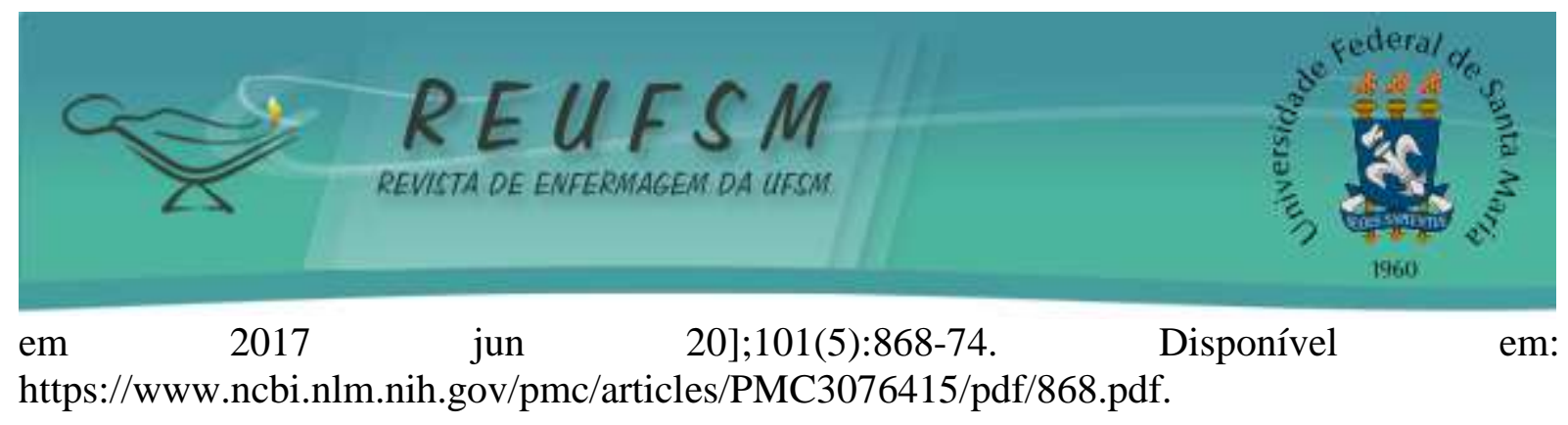

23. Oliveira AMS, Menezes TMO. A enfermeira no cuidado ao idoso na estratégia saúde da família: sentidos do vivido. Rev Enferm UERJ [Internet]. 2014 [acesso em 2017 set 06];22(4):513-8. Disponível em: http://www.facenf.uerj.br/v22n4/v22n4a13.pdf.

24. Nunes APN, Barreto SM, Gonçalves LG. Relações sociais e autopercepção da saúde: Projeto Envelhecimento e Saúde. Rev Bras Epidemiol [Internet]. 2012 [acesso em 2017 set 05];15(2):415-28. Disponível em: http://www.scielo.br/pdf/rbepid/v15n2/19.pdf.

25. Wichmann FMA, Couto AN, Areosa SVC, Montañés MCM. Grupos de convivência como suporte ao idoso na melhoria da saúde. Rev Bras Geriatr Gerontol [Internet]. 2013 [acesso em 2017 jun 20];16(4):821-32. Disponível em: http://www.scielo.br/pdf/rbgg/v16n4/1809-9823-rbgg-16-04-00821.pdf.

Data de submissão: 20/06/2017

Data de aceite: 29/10/2018

Autor correspondente: Mariangela Uhlmann Soares

E-mail: mariangela.soares@gmail.com

Endereço: R. São Leopoldo, 924, Laranjal. Pelotas/RS. CEP 96090-700 